\title{
O IMPACTO DAS VARIÁVEIS “ORIGEM DO TRÁFEGO" E “PÁGINA DE DESTINO" NAS MÉTRICAS DE ENGAJAMENTO EM SITES DE NOTÍCIAS: ANÁLISE DO SITE D’O GLOBO
}

Augusto de Freitas Lohmann

Doutorando em Design - Esdi/UERJ

augustolohmann@gmail.com

Resumo: $O$ artigo apresenta os resultados da análise das métricas do Google Analytics do site d'O Globo (www.oglobo.com.br) entre o período de 15/10/2015 a 15/01/2016. A análise teve por objetivo identificar o impacto das variáveis "origem do tráfego" e "página de destino" em um maior ou menor engajamento dos usuários em sites de notícias, a partir dos padrões identificados nas métricas d'O Globo. Como resultados, observou-se que a "origem do tráfego" tem pouca influência no engajamento dos usuários, e que a experiência oferecida na "página de destino" é o fator que promove o maior engajamento do usuário com o site de notícias.

Palavras-chave: métricas, engajamento, notícias, origem do tráfego, página de destino.

\begin{abstract}
The article presents the analysis of metrics of $O$ Globo site (www.oglobo.com.br) for the period from 10/15/2015 to 15/01/2016. This analysis aimed to identify the impact of variables "origin referrer" and "destination page" to a greater or lesser engagement of users on news sites, based on the metrics of O Globo. As a result, it was observed that the "origin referrer" has little influence on user engagement, and that the experience offered in the "destination page" is the factor that promotes greater engagement.
\end{abstract}

Keywords: metrics, engagement, news, origin referrer, destination page 


\section{INTRODUÇÃO}

Nos últimos anos, os acessos a sites de notícias originados a partir de buscadores, redes sociais ou outras fontes externas, quando consolidados, se tornaram maioria em relação aos acessos iniciados via tráfego direto. Ao contrário do que acontece no tráfego direto, onde grande parte dos usuários iniciam a navegação pela homepage do site, no acesso originado por fontes externas a maior parte dos usuários tende a iniciar sua navegação diretamente pelas páginas de conteúdo (usualmente a matéria jornalística). Dessa forma, pode-se concluir que as homes dos sites de notícias vêm perdendo força em sua função de porta de entrada, ao passo que cada vez mais a primeira experiência do usuário com o site se dá justamente pela página do conteúdo.

Entretanto, métricas e pesquisas apontam que o usuário que acessa um site de notícias a partir de fontes externas tende a ser significativamente menos engajado com o produto noticioso do que os que acessam via tráfego direto. Tais métricas ainda são de fundamental importância na captação de receita por parte dos sites de notícias, o que constitui a melhora dessas métricas como um dos grandes desafios atuais para o modelo do jornalismo digital.

Nesse artigo, será apresentada uma análise feita sobre as métricas de utilização do site d'O Globo (www.oglobo.com.br), utilizando a ferramenta Google Analytics. A análise objetiva identificar: 1) o impacto da origem do tráfego (ou seja, o contexto imediatamente anterior ao acesso ao site de notícias) no engajamento do usuário com o produto noticioso; e 2) a diferença no engajamento quando o tipo de página de destino em que se inicia a navegação é uma home ou uma página de conteúdo. Por exemplo, um usuário que acessa o site d'O Globo a partir de um link compartilhado no Facebook, ou de uma pesquisa no Google, se comporta de maneira diferente do usuário que acessa via tráfego direto simplesmente pelo contexto diferente em que se originou a experiência? 0 fator de impacto nas métricas de engajamento está na origem do tráfego (ou seja, no contexto imediatamente anterior ao acesso), na página de destino em que se inicia a experiência (ou seja, na experiência de uso que é apresentada imediatamente após o acesso ao site de notícias), ou em ambos? A análise e seus resultados serão parte integrante da tese de doutorado do autor (em curso), auxiliando na identificação de elementos do design de experiência que auxiliem na melhora das métricas de engajamento de usuários de sites de notícias.

\section{DESENVOLVIMENTO}

Em pesquisa realizada pelo The Pew Research Center's Project for Excellence in Journalism (figura 1), observa-se que apenas $36 \%$ dos usuários acessam sites de notícias via tráfego direto, enquanto os $64 \%$ restantes se originam por origens externas. 


\section{Social media is not an overwhelming driver of news (yet) \\ Percent of U.S. adults who get news on \\ any digital device very often.. \\ ...through Facebook \\ or Twitter \\ $9 \%$ \\ recommendations \\ by going directly \\ to news websites \\ or apps \\ 36 \\ ...by using key \\ word search \\ 32 \\ through a news \\ organizing web \\ site or app \\ 29 \\ $\mathrm{N}=3,016$ \\ REW PESEAPCH CENTERS PROIECT FOR EXCELIENCE IN JOURNALSM \\ 2012 STATE OF THE NEWS MEDIA}

Figura 1 - Percentual de adultos americanos que acessa notícias em dispositivos digitais por diferentes origens.

Fonte: "MITCHELL, Amy; ROSENTIEL, Tom. 2013".

Já no aspecto do engajamento dos usuários em sites de noticias, outra pesquisa realizada em 2013 pelo mesmo The Pew Research Center's Project for Excellence in Journalism (figura 2) aponta que o engajamento de quem acessa diretamente um site de notícias pela homepage é significativamente maior do que de usuários cuja origem do tráfego se dá a partir do Facebook ou de mecanismos de busca.

\section{Referral Traffic to News Sites}

Average monthly...

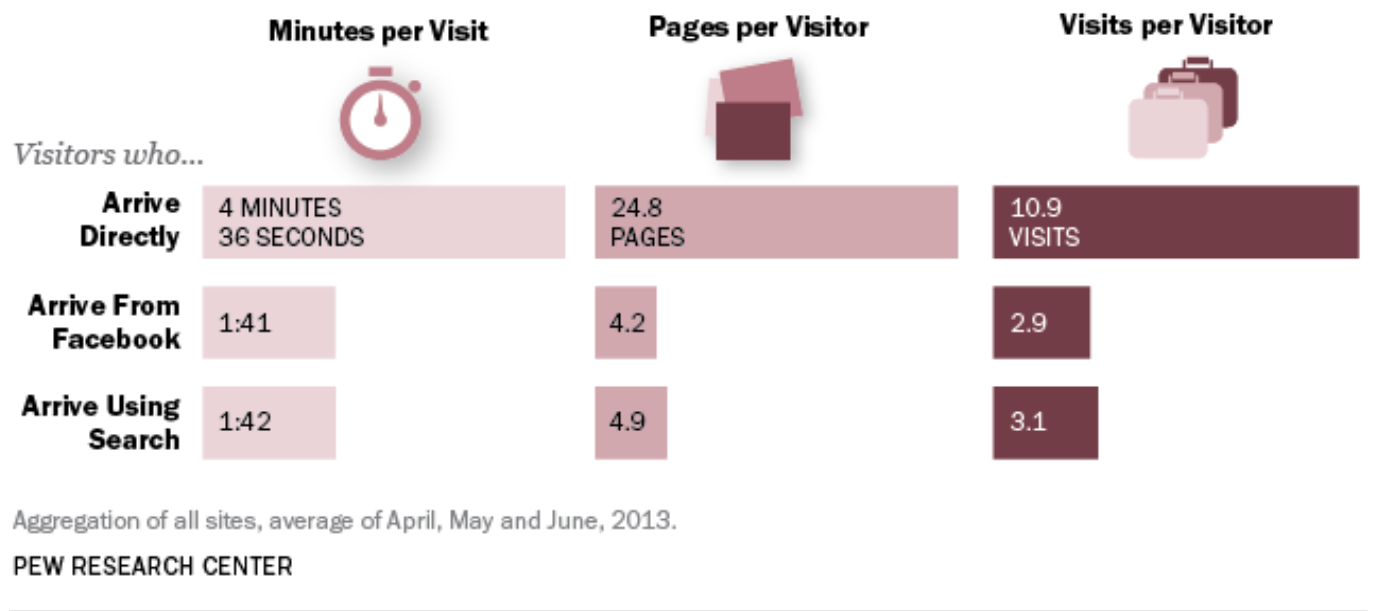

Figura 2 - Pesquisa do Pew Research Center comparando métricas de usuários que acessam por tráfego direto ou vindo de redes sociais e buscadores.

Fonte: "HAILE, Tony. 2014". 
Dessa maneira, em um cenário onde é cada vez menor propocionalmente o tráfego direto aos sites de notícias em comparação ao acesso via redes sociais, buscadores e outras fontes externas, o baixo engajamento vem se tornando um problema crescente para os sites de notícias. Atualmente a maior parte dos sites de notícias do Brasil e do Mundo vêm buscando formas de estimular a navegação de seus usuários e a melhora na experiência de uso do produto noticioso como um todo, buscando a melhora nas métricas de utilização.

A pesquisa apresentada nesse artigo tem por objetivo identificar o impacto de duas variáveis da experiência de uso dos sites de notícias em um maior ou menor engajamento por parte do usuário: 1) Origem do Tráfego (de onde veio o usuário antes de acessar o site); e 2) Página de destino (qual a primeira página acessada pelo usuário no site). Ou seja, o contexto imediatamente anterior ao acesso impacta na sequência da experiência de uso no site de notícias, ou a própria experiência oferecida pelo site é o que promove um maior engajamento? A avaliação do impacto dessas variáveis é de fundamental importância na definição de soluções específicas que estimulem a melhora nas métricas de engajamento de usuários de sites de notícias.

Como metodologia de pesquisa, inicialmente foi feito um levantamento e análise das métricas de utilização do site d'O Globo (Google Analytics) em um período de 3 meses (entre 15/10/2015 e 15/01/2016). Tal período foi definido visando evitar possíveis distorções/variações que poderiam se apresentar em períodos mais curtos de tempo. As métricas selecionadas para a análise foram: taxa de rejeição (percentual de usuários que abandona o site sem navegar por outras páginas além da página de destino inicial), páginas por sessão (média do número de páginas visitadas em uma sessão) e duração média por sessão (média do tempo de navegação em cada sessão no site). Essas métricas foram selecionadas por representarem indicadores de engajamento do usuário com o site.

Após a definição do recorte de tempo e a seleção das métricas, as variáveis "origem do tráfego" e "página de destino" foram utilizadas na segmentação em múltiplos cenários que permitissem a avaliação do impacto dessas variáveis nas métricas de engajamento. Como "origem do tráfego", foram selecionadas as 4 origens mais representativas no contexto do site d'O Globo, como representado na figura 3: 1) Globo.com; 2) Google; 3) Facebook e 4) Tráfego Direto 
Figura 3 - Origem do tráfego no site d’O Globo (número de sessões).

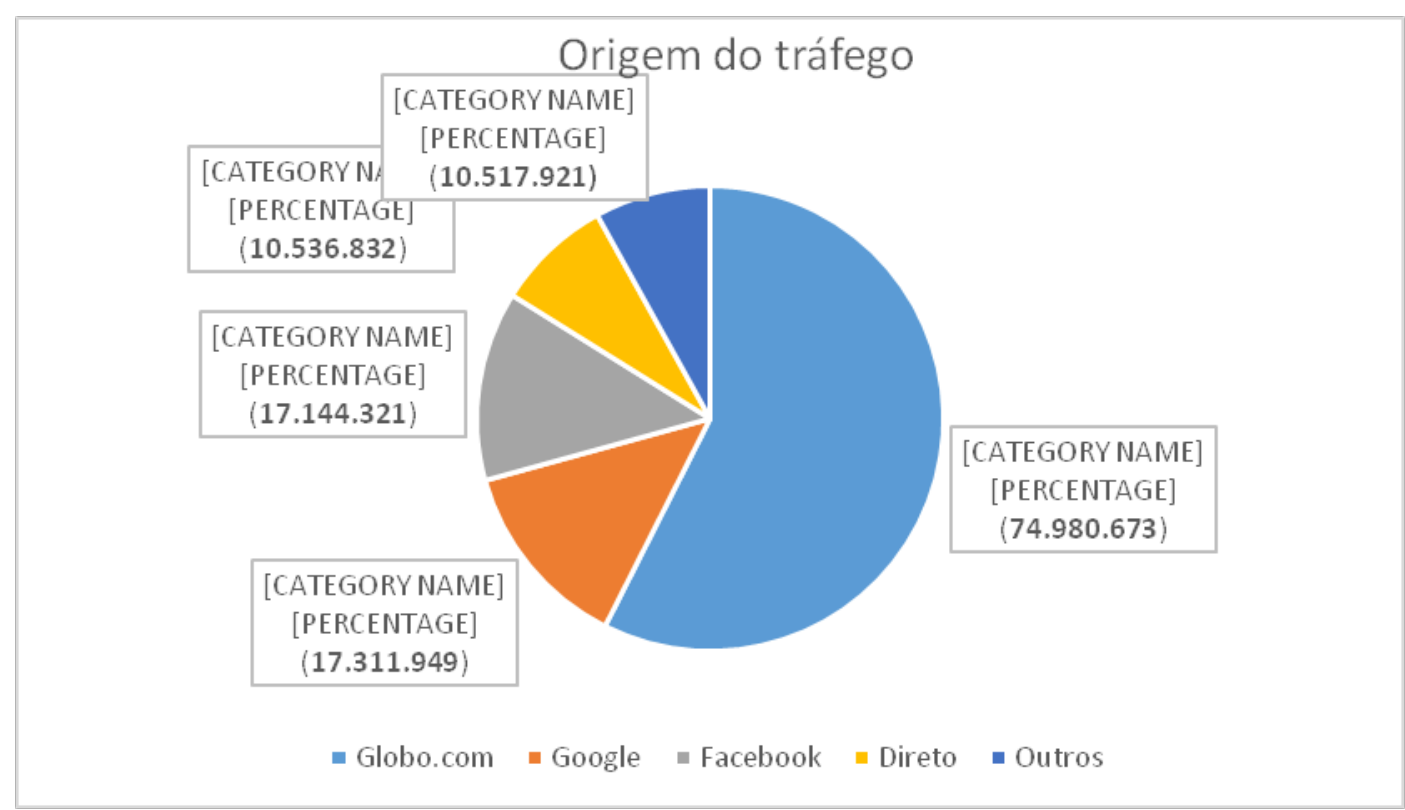

Fonte: "Elaborado pelo autor, com base na pesquisa realizada".

Já os resultados observados para a variável "página de destino" foram agrupados em 2 tipos principais: 1) Homes (quando a primeira página acessada na sessão é uma home do site) e 2) Páginas de Conteúdo (quando a primeira página acessada na sessão é uma página de conteúdo).

A seguir, são apresentadas as métricas obtidas a partir da construção de múltiplos cenários envolvendo o cruzamento das variáveis definidas.

\subsection{Análise de métricas do site desktop d’O Globo (oglobo.com.br)}

A tabela 1 apresenta uma análise inicial das 3 métricas selecionadas (taxa de rejeição, páginas por sessão e duração média da sessão), segmentada pelos 4 parâmetros da variável "origem do tráfego" (direto, Facebook, Google e Globo.com) no site d'O Globo.

Tabela 1 - Métricas por origem do tráfego

\begin{tabular}{llll}
\hline Origem do Tráfego & Taxa de rejeição & Páginas p/ sessão & Duração média da sessão \\
\hline Direto & $51,76 \%$ & 2,87 & $11: 45$ \\
\hline Facebook & $78,43 \%$ & 1,44 & $2: 35$ \\
\hline Globo.com & $66,09 \%$ & 1,59 & $2: 34$ \\
\hline Google & $52,58 \%$ & 2,80 & $9: 05$ \\
\hline
\end{tabular}

Fonte: Métricas do Google analytics do site oglobo.com.br, no período de 3 meses (15/10/2015 a 15/01/2016).

Como é possível observar, o acesso via tráfego direto é o que apresenta os melhores resultados (menor taxa de rejeição, maior quantidade de páginas por sessão e mais tempo de navegação por sessão). Já o acesso originado pelo Facebook 
apresenta as piores taxas de engajamento, com uma taxa de rejeição quase $30 \%$ maior em valores absolutos, com a metade do número de páginas por sessão e com um tempo de navegação de cerca de $20 \%$ do registrado nos acessos via tráfego direto.

Tais resultados corroboram a pesquisa apresentada anteriormente (figura 2), confirmando que no site d'O Globo o acesso via tráfego direto também apresenta métricas de engajamento melhores do que as demais origens de tráfego analisadas.

A partir dos resultados apresentados na tabela 1, seria plausível concluir que o Facebook como origem do tráfego em um site de notícias seria um fator de impacto no (baixo) engajamento. Entretanto, faz-se necessário um aprofundamento no estudo das métricas a fim de compreender se as diferentes origens de tráfego são realmente a causa de um maior ou menor engajamento por parte dos usuários, ou se outros fatores impactam nessa questão.

Para isso, o próximo passo foi aprofundar a análise das métricas, mantendo a segmentação por cada uma das origens de tráfego (direto, facebook, globo.com e google) mas incluindo a variável "página de destino". Conforme a metodologia apresentada, foram definidos 2 parâmetros: "homes" (quando a página de destino é uma homepage) e "conteúdo" (quando a página de destino é um conteúdo).

A tabela 2 apresenta as métricas "taxa de rejeição", páginas por sessão" e "duração média da sessão" quando o acesso se origina via tráfego direto, segmentado pela variável "página de destino" (por homes X conteúdos).

Tabela 2 - Tráfego Direto (site desktop O Globo)

\begin{tabular}{lll}
\hline & Página de destino: Homes & Página de destino: Conteúdos \\
\hline Taxa de Rejeição & $39,62 \%$ & $61,09 \%$ \\
\hline Páginas por Sessão & 3,59 & 1,70 \\
\hline Duração Média da Sessão & $18: 10$ & $2: 35$
\end{tabular}

Fonte: Métricas do Google analytics do site oglobo.com.br, no período de 3 meses (15/10/2015 a 15/01/2016)

A tabela 3 apresenta as métricas "taxa de rejeição", páginas por sessão" e "duração média da sessão" quando o acesso se origina via Facebook, segmentando pela variável "página de destino" (por homes X conteúdos).

Tabela 3 - Tráfego via Facebook (site desktop O Globo)

\begin{tabular}{lll}
\hline & Página de destino: Homes & Página de destino: Conteúdos \\
\hline Taxa de Rejeição & $36,64 \%$ & $63,47 \%$ \\
\hline Páginas por Sessão & 3,92 & 1,32 \\
\hline Duração Média da Sessão & $24: 13$ & $1: 23$ \\
& & \\
\hline
\end{tabular}

Fonte: Métricas do Google analytics do site oglobo.com.br, no período de 3 meses (15/10/2015 a 15/01/2016). 
A tabela 4 apresenta as métricas "taxa de rejeição", páginas por sessão" e "duração média da sessão" quando o acesso se origina via Globo.com, segmentando pela variável "página de destino" (por homes X conteúdos).

Tabela 4 - Tráfego via Globo.com (site desktop O Globo).

\begin{tabular}{lll}
\hline & Página de destino: Homes & Página de destino: Conteúdos \\
\hline Taxa de Rejeição & $36,02 \%$ & $55,99 \%$ \\
\hline Páginas por Sessão & 4,03 & 1,41 \\
\hline Duração Média da Sessão & $20: 38$ & $1: 43$
\end{tabular}

Fonte: Métricas do Google analytics do site oglobo.com.br, no período de 3 meses (15/10/2015 a 15/01/2016).

A tabela 5 apresenta as métricas "taxa de rejeição", páginas por sessão" e "duração média da sessão" quando o acesso se origina via Google, segmentando pela variável "página de destino" (por homes $\mathrm{X}$ conteúdos).

Tabela 5 - Tráfego via Google (site desktop O Globo)

\begin{tabular}{lll}
\hline & Página de destino: Homes & Página de destino: Conteúdos \\
\hline Taxa de Rejeição & $36,02 \%$ & $67,47 \%$ \\
\hline Páginas por Sessão & 3,89 & 1,51 \\
\hline Duração Média da Sessão & $18: 11$ & $1: 35$
\end{tabular}

Fonte: Métricas do Google analytics do site oglobo.com.br, no período de 3 meses (15/10/2015 a 15/01/2016).

A seguir, serão apresentados gráficos que consolidam as métricas apresentadas, bem como a análise dos resultados.

\section{DISCUSSÃO}

O gráfico da figura 4 consolida a métrica "taxa de rejeição" em um comparativo homes $\mathrm{X}$ conteúdos, por cada uma das 4 origens de tráfego. Como pode ser observado, independente da origem via tráfego direto, Facebook, Globo.com ou Google, a taxa de rejeição de quem inicia seu acesso por uma home page apresenta percentuais bastante parecidos. Da mesma forma, quando o acesso se inicia por páginas de conteúdo, a taxa de rejeição é consistentemente elevada (ou seja, muitos usuários abandona rapidamente o site) e muito parecida entre si, independente da origem do tráfego. 


\section{TAXA DE REJEIÇÃO (\%)}

ORIGEM DO TRÁFEGO

\begin{tabular}{l|r|l} 
Direto & $39,62 \%$ & $61,09 \%$ \\
\hline Facebook & $36,64 \%$ & $63,47 \%$ \\
\hline Globo.com & $36,02 \%$ & $55,99 \%$ \\
\hline Google & $36,02 \%$ & $67,47 \%$
\end{tabular}

Figura 4 - Métrica "Taxa de rejeição" do site d'O Globo por acessos iniciados via homes e por acessos iniciados por páginas de conteúdo, segmentados pela "origem do tráfego".

Fonte: "Elaborado pelo autor, com base na pesquisa realizada".

Nesse caso, é possível observar que mesmo acessando via tráfego direto (onde, a princípio, o engajamento seria sempre maior), a taxa de rejeição é muito elevada quando a página de destino é uma página de conteúdo. Da mesma forma, quando o acesso se dá via Facebook (onde o engajamento seria sempre menor), a taxa de rejeição é muito baixa quando o destino do usuário é uma home, e não uma página de conteúdo.

Já o gráfico da figura 5 consolida a métrica "páginas por sessão" em um comparativo homes $\mathrm{X}$ conteúdos, por cada uma das 4 origens de tráfego. Novamente observa-se que independente da origem via tráfego direto, Facebook, Globo.com ou Google, o número de páginas por sessão de quem inicia seu acesso por uma home apresenta números bastante parecidos entre si. Da mesma forma, quando o acesso se inicia por páginas de conteúdo, o número de páginas por sessão é consideravelmente mais baixo (menos da metade) e também muito parecida entre si, independente da origem do tráfego.

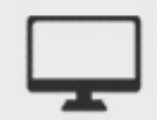

ORIGEM DO TRÁFEGO

\begin{tabular}{l|c|c} 
Direto & 3,59 & 1,70 \\
\hline Facebook & 3,92 & 1,32 \\
\hline Globo.com & 4,03 & 1,41 \\
\hline Google & 3,89 & 1,51 \\
\hline
\end{tabular}

Figura 5 - Métrica "páginas por sessão" do site d’O Globo por acessos iniciados via homes e por acessos iniciados por páginas de conteúdo, segmentados pela "origem do tráfego". Fonte: "Elaborado pelo autor, com base na pesquisa realizada". 
A exemplo do que foi observado na métrica anterior, não é a origem do acesso que resulta em um maior engajamento, mas sim a página de destino (homes promovendo maior engajamento em relação a conteúdos).

No gráfico apresentado na figura 6, a métrica "duração média da sessão" é apresentada em um comparativo homes $X$ conteúdos, por cada uma das 4 origens de tráfego. Mais uma vez, independente da origem via tráfego direto, Facebook, Globo.com ou Google, a duração média da sessão de quem inicia seu acesso por uma home apresenta números bastante parecidos entre si. Quando o destino é uma página de conteúdo, a duração média da sessão é em média 15 vezes menor, mas também muito parecida entre si, independente da origem do tráfego.

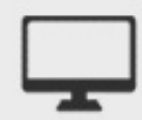

\section{DURAÇÃO MÉDIA DA SESSÃO (mm:ss)}

ORIGEM DO TRÁFEGO

\begin{tabular}{l|c|c} 
Direto & $18: 10$ & $2: 35$ \\
\hline Facebook & $24: 13$ & $1: 23$ \\
\hline Globo.com & $20: 38$ & $1: 43$ \\
\hline Google & $18: 11$ & $1: 35$
\end{tabular}

Figura 6 - Métrica “Duração média da sessão" do site d'o Globo por acessos iniciados via homes e por acessos iniciados por páginas de conteúdo, segmentados pela "origem do tráfego".

Fonte: "Elaborado pelo autor, com base na pesquisa realizada".

Como ressaltado na análise das 2 métricas anteriores, a origem do acesso pouco influencia em maior ou menor engajamento, e novamente a página de destino é a variável preponderante na diferença das métricas.

Com base nessa análise, apesar de ser verdadeira a afirmação de que origens de tráfego diferentes apresentam métricas de engajamento diferentes, e que o acesso via tráfego direto apresenta métricas muito melhores em relação às demais origens de tráfego, a causa desse engajamento não está na origem, ou seja, no contexto anterior ao acesso ao site de notícias. Ao segmentar os acessos pela va riável "página de destino" ser uma home ou um conteúdo, consistentemente observa-se que uma sessão iniciada pelas homes apresenta métricas melhores do que uma sessão iniciada por conteúdos.

\section{CONCLUSÃO}

Dessa forma, conclue-se que o fator de impacto no engajamento do usuário de um site de notícias está na muito mais na experiência que é oferecida pela "página de destino" pela qual o usuário tem o primeiro contato com o site. As homes, apesar de cada vez serem menos acessadas, oferecem uma experiência de uso que diminui a 
taxa de rejeição e amplia o número de páginas acessadas e a duração da navegação dos usuários. Em um caminho oposto, as páginas de conteúdo são cada vez mais porta de entrada de quem acessa sites de notícias, mas promovem pouco engajamento para a sequência da navegação.

É nesse cenário que se torna de fundamental importância para os sites de notícias o entendimento de que as páginas de conteúdos adquirem o papel de landing pages, e que a manutenção do engajamento de seus usuários passa pelo design de uma experiência de uso que contemple essa mudança de comportamento. Um dos caminhos que se apresenta é o de incutir nas páginas de conteúdo elementos característicos das homes, com ênfase na oferta de uma experiência de uso mais satisfatória e que incentive naturalmente a permanência dos usuários no site de notícias.

\section{REFERÊNCIAS}

HAILE, Tony. What You Think You Know About the Web Is Wrong. Time.com, 2014. Disponível na internet por http em: http://time.com/12933/what-you-think-you-knowabout-the-web-is-wrong Acesso em 19 nov. 2015

MITCHELL, Amy; ROSENTIEL, Tom. What Facebook and Twitter Mean for News. 2013. Disponível na internet por http em: <http://stateofthemedia.org/2012/mobile-devicesand-news-consumption-some-good-signs-for-journalism/what-facebook-and-twittermean-for-news> Acesso em 10 abr. 2015. 